\title{
Patterns of Multidisciplinary Care in the Management of Non- metastatic Invasive Breast Cancer in the United States Medicare Patient
}

\author{
Thomas M. Churilla, MD*, Brian L. Egleston, $\mathrm{PhD}^{\dagger}$, Colin T. Murphy, MD*, Elin R. Sigurdson,

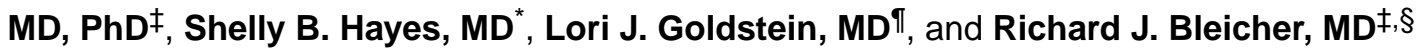 \\ "Department of Radiation Oncology, Fox Chase Cancer Center, Philadelphia, PA \\ tDepartment of Biostatistics, Fox Chase Cancer Center, Philadelphia, PA \\ ‡Department of Surgical Oncology, Fox Chase Cancer Center, Philadelphia, PA \\ IDepartment of Medical Oncology, Fox Chase Cancer Center, Philadelphia, PA
}

\begin{abstract}
PURPOSE-Multidisciplinary care (MDC) in managing breast cancer is resource-intensive and growing in prevalence anecdotally, although care patterns are poorly characterized. We sought to determine MDC patterns and effects on care in the United States Medicare patient.
\end{abstract}

\begin{abstract}
METHODS—Patients diagnosed with non-metastatic invasive breast cancer from 1992-2009 were reviewed using the Survival, Epidemiology, and End Results (SEER)-Medicare linked dataset. MDC was defined as a post-diagnosis, preoperative visit with a surgical, medical, and radiation oncologist. Same day-MDC $\left(\mathrm{MDC}_{\mathrm{SD}}\right)$ was the $\mathrm{MDC}$ subset having all three visits on one date.
\end{abstract}

RESULTS-Among 88,865 patients, MDC was utilized in 2.9\%, with $12 \%$ of these having $\mathrm{MDC}_{\mathrm{SD}}$. MDC use did not vary by stage, but MDC patients were more likely to be younger, black, receive lumpectomy, have fewer nodes examined, and receive radiotherapy. $\mathrm{MDC}_{\mathrm{SD}}$ patients were more likely than non-MDC patients to be black, receive mastectomy, and receive radiotherapy. $\mathrm{MDC}$ and $\mathrm{MDC}_{\mathrm{SD}}$ use increased over time and varied by geographic region, with rural patients less likely to receive $\mathrm{MDC}(\mathrm{OR} 0.54[95 \%$ CI $0.45-0.65])$ and $\mathrm{MDC}_{\mathrm{SD}}(\mathrm{OR} 0.32[95 \%$ CI 0.19

\footnotetext{
$\S^{\S}$ Corresponding Author: Richard J. Bleicher, MD, Fox Chase Cancer Center, 333 Cottman Avenue, Room C-308, Philadelphia, PA 19111, 215-728-2596 Voice, 215-728-2773 Facsimile, richard.bleicher@ fccc.edu.

Presented, in part, at the 2015 San Antonio Breast Cancer Symposium, San Antonio, TX.

Author Contributions:

Planning: T Churilla, B Egleston, R Bleicher

Conduct: T Churilla, B Egleston, C Murphy, E Sigurdson, S Hayes, L Goldstein, R Bleicher.

Reporting: T Churilla, B Egleston, C Murphy, E Sigurdson, S Hayes, L Goldstein, R Bleicher.

Guarantor of overall content: R Bleicher

Compliance with Ethical Standards:

Conflicts of Interest: The authors have no conflicts of interest to disclose.

Ethical approval: All procedures performed in studies involving human participants were in accordance with the ethical standards of the institutional and/or national research committee and with the 1964 Helsinki declaration and its later amendments or comparable ethical standards.

The authors report no other conflicts of interest.
} 
0.54]). Radiotherapy after breast conserving surgery, used in $86.2 \%$ of non-MDC patients, was administered to $89.0 \%$ of $\mathrm{MDC}(\mathrm{p}<0.001)$ and $92.6 \%$ of $\mathrm{MDC}_{\mathrm{SD}}(\mathrm{p}=0.096)$ patients. Postmastectomy radiotherapy was administered in $52.0 \%$ of non-MDC patients, $63.8 \%$ of $\operatorname{MDC}(\mathrm{p}=0.050)$, and $89.1 \%$ of $\mathrm{MDC}_{\mathrm{SD}}(\mathrm{p}=0.011)$ after propensity score adjustment.

CONCLUSION-While increasing, few Medicare patients undergo $\mathrm{MDC}$ and $\mathrm{MDC}_{\mathrm{SD}}$ is rare. MDC may improve quality and $\mathrm{MDC}_{\mathrm{SD}}$ should be considered for patient convenience. While not yet widespread, efforts should integrate $\mathrm{MDC}$ and $\mathrm{MDC}_{\mathrm{SD}}$ across the U.S.

\section{Keywords}

Multidisciplinary care; medical oncology; surgical oncology; radiation oncology; breast cancer

\section{INTRODUCTION}

Care of the patient with breast cancer has increased in complexity over the past several decades because of data demonstrating survival benefits from multimodal therapy.[1,2] Consequently, care of the oncology patient has evolved to coordinate these new treatment paradigms for efficiency and patient convenience, and the term multidisciplinary care (MDC) frequently refers to coordination among specialties. Despite its perceived benefits, the patterns of multidisciplinary care in the United States have not been previously investigated to our knowledge. There is no standard definition for MDC, and no reference for what can be considered standard practice in the multidisciplinary treatment of the breast cancer patient.

Treatment of breast cancer represents an ideal target for MDC, because of the interdisciplinary paradigms used, as well as the interdependency of the therapeutic modalities used in treatment. Despite favorable perceptions by patients and providers, there is also surprisingly little data demonstrating improvements in outcomes resulting from MDC use, and no data investigating the benefits to breast cancer patients on a national level.[3-11]

The National Accreditation Program for Breast Centers (NAPBC) of the American College of Surgeons has identified formal interdisciplinary team management involving consultations with multiple specialists as a standard component for breast cancer centers, although this does not specify the timing of the care that should be provided.[12] As the concept of MDC has now become considered as a quality indicator, we sought to examine the incidence of MDC involving formal consultations provided preoperatively to determine if this is standard practice, to evaluate factors predictive of its use in managing localized breast cancer for the United States Medicare population, and to see if quality metrics quantifiably improve with its use.

\section{METHODS}

After IRB and NCI approval, we reviewed cases from 1992-2009 from the National Cancer Institute (NCI) Surveillance, Epidemiology, and End Results (SEER)-Medicare linked database. Women $\Varangle 65$ years of age have the highest breast cancer incidence, and unlike SEER or the National Cancer Database, the SEER-Medicare linked dataset is one of the few 
large national datasets with sufficient granularity from its claims data[13] to determine the national patterns of care pertinent to this study.

We included patients with invasive, non-metastatic, non-inflammatory breast cancer. Inclusion and exclusion criteria are outlined in Figure 1. The date of diagnosis was defined as the first biopsy date occurring in the same month or month after the SEER clinical diagnosis date. We included patients who underwent surgery for breast cancer within 6 months after the date of diagnosis. We excluded patients who received neoadjuvant chemotherapy for treatment homogeneity and to more accurately assess who was seeing all 3 specialties even when they did not necessarily require it for therapy preoperatively.

\section{Patterns of Care}

MDC was defined as a visit with a surgeon, a medical oncologist, and a radiation oncologist all preoperatively on the same or different dates. We queried new patient/consultations or follow-up visits occurring within 2 months of the date of diagnosis for general surgery or surgical oncology, medical oncology or hematology/oncology, and radiation oncology. Health Care Financing Administration (HCFA) provider-specific codes were used to identify provider type. Supplemental Tables 1 and 2 list the Current Procedural Terminology (CPT) and line HCFA codes used to abstract the relevant provider visits. We limited to consultations within a 2 month time frame to query for a pattern of coordinated upfront provider visits. Two separate analyses were performed: one evaluating all MDC patients (MDC), and the second characterizing the subset of patients who saw all three specialties on the same day (MDC same date, $\mathrm{MDC}_{\mathrm{SD}}$ ).

\section{Treatment Quality}

To measure the impact of MDC on quality metrics, we assessed patterns of care similar to American College of Surgeons' Commission on Cancer[14] and NAPBC[15] quality measures.[14] The following measures were evaluated: rate of breast conserving surgery versus mastectomy for stage 0 , I and II breast cancer, adjuvant radiation therapy following breast conserving surgery, adjuvant post-mastectomy radiation therapy for patients with either T3/T4 primaries or $\geq 4$ positive lymph nodes, adjuvant chemotherapy use among node positive patients, and the use of sentinel node biopsy. Supplemental Table 3 lists the CPT codes used to define the quality metrics.

\section{Statistical Analyses}

We used T-tests and chi-squared tests to compare unadjusted differences. We used a multiple logistic regression to investigate the predictors of breast conservation therapy. To investigate adjusted differences in quality metrics between MDC groups, we used multiple logistic regressions with propensity score based-weighting (i.e. inverse probability of treatment weighting [IPTW]).[16, 17] We used multiple logistic regression models to estimate the propensity score. In the propensity score models for the quality metrics, we included metropolitan status (e.g. rural for large metropolitan area), SEER geographic region, year of diagnosis, nodes examined, nodes positive, histology, Elixhauser score, [18, 19] Charlson score,[20] percentage of residents in case's census tract with less than a high school education, median income in case's census tract, age at diagnosis, tumor size, sequence of 
breast cancer (i.e. of all cancer diagnoses that a person had, the order in which the first breast cancer occurred), marital status, race, AJCC tumor stage, and grade of tumor.

To examine adjusted survival differences among groups, we used propensity-score based weighted Cox proportional hazards regressions for overall survival, and Fine and Gray proportional hazards regressions for cause specific survival.[21] In addition to the covariates used for the quality metric analyses, we also included indicator variables denoting surgery (BCT versus mastectomy), radiotherapy, and chemotherapy use. In the propensity score models, we incorporated continuous variables through the use of restricted cubic splines with two knots.[22] We examined the propensity score-based weighted differences between MDC groups to ensure that the propensity scores appropriately balanced the data between arms, on average, and used interaction terms in the propensity score model, as necessary, to ensure that all covariates were balanced. We used bootstrap standard errors that accounted for estimation of the propensity score in the weighted models, and set the criteria for statistical significance as $\mathrm{p} \leq 0.05$.

We performed sensitivity analyses excluding patients over age 80 for the quality metrics analysis, and excluding patients diagnosed before the year 2000 for the sentinel lymph node analysis. We used STATA version 13 (StataCorp, College Station, Texas) for analyses.

\section{RESULTS}

There were 88,865 Medicare patients after all exclusions (Figure 1) with 2,538 (2.8\%) patients having MDC with 357 (14.1\%) of these having their surgical, radiation oncology, and medical oncology appointment on the same date $\left(\mathrm{MDC}_{\mathrm{SD}}\right) .1,098$ patients who received neoadjuvant chemotherapy were excluded, of which $12.1 \%$ received MDC, and $0.6 \%$ received $\mathrm{MDC}_{\mathrm{SD}}$. Baseline patient and general treatment characteristics for MDC and $\mathrm{MDC}_{\mathrm{SD}}$ are listed in Table 1. The practice of MDC increased over time for both MDC and $\mathrm{MDC}_{\mathrm{SD}}$ as illustrated in Figure 2 ( $\mathrm{p}<0.001$ for both trends).

\section{Patterns of Care}

Table 2 outlines the clinical and demographic variables associated with the practice of MDC and $\mathrm{MDC}_{\mathrm{SD}}$. Younger patients were more likely to receive MDC. The practice of MDC did not vary significantly by AJCC stage, patient comorbidity, tumor grade, or histology. The incidence of MDC varied according to region, however, with a 22.5 fold decrease in the rate of $\mathrm{MDC}_{\mathrm{SD}}$ in the south compared to the midwest. Patients living in more sparsely populated areas were significantly less likely to receive $\mathrm{MDC}$ and $\mathrm{MDC}_{\mathrm{SD}}$. Black patients more frequently underwent $\mathrm{MDC}$ and $\mathrm{MDC}_{\mathrm{SD}}$, while patients living in counties with lower education rates were less likely to undergo $\mathrm{MDC}$ and $\mathrm{MDC}_{\mathrm{SD}}$.

\section{Treatment Quality}

Table 3 demonstrates unadjusted and propensity score-based weighted estimates of quality measures associated with the practice of MDC. Overall, the rates of sentinel node biopsy were similar among the non-MDC, $\mathrm{MDC}$ and $\mathrm{MDC}_{\mathrm{SD}}$ groups. Administration of adjuvant radiation following breast conserving surgery was significantly greater in the setting of $\mathrm{MDC}$ and $\mathrm{MDC}_{\mathrm{SD}}$. Among patients having indications for post-mastectomy radiotherapy 
( $\mathrm{T} 3$ or $\mathrm{T} 4$ tumors, or $\geq 4$ positive lymph nodes), absolute rates of adjuvant radiation therapy were $11.8 \%$ (MDC) and $37.3 \%\left(\mathrm{MDC}_{\mathrm{SD}}\right)$ higher than non-MDC patients with borderline statistical significance. Use of adjuvant chemotherapy among node-positive patients was significantly more frequent among the $\mathrm{MDC}_{\mathrm{SD}}$ cohort with an absolute increase of $4.4 \%$ compared to non-MDC. Finally, patients having MDC and $\mathrm{MDC}_{\mathrm{SD}}$ experienced significantly longer mean times between diagnosis to surgery in propensity matched comparisons with an increase of 12.6 days and 7.8 days, respectively.

A sensitivity analysis excluding patients diagnosed before the year 2000 did not significantly change results for the sentinel lymph node analysis. A second sensitivity analysis excluding patients over the age of 80 also did not significantly change results for the remaining quality metrics analyses.

\section{Outcomes}

After propensity score adjustment, including surgery type, adjuvant chemotherapy use, and administration of adjuvant radiation therapy, overall survival (OS) estimates for MDC were HR $[95 \% \mathrm{CI}]=0.86$ [0.73-1.02], $\mathrm{p}=0.092$, while breast cancer-specific survival estimates had a sHR [95\% CI] $=0.80[0.63-1.02], \mathrm{p}=0.073$. Among the $\mathrm{MDC}_{\mathrm{SD}}$ cohort, OS estimates were HR [95\% CI] $=0.57$ [0.29-1.12], $\mathrm{p}=0.101$, while breast cancer-specific survival estimates were sHR [95\% CI] $=1.06$ [0.28-4.07], $p=0.934$. Supplemental Figure 1 shows the adjusted overall and cause-specific survival curves for both cohorts.

\section{DISCUSSION}

Despite this growing perception that formal preoperative consultation with multiple oncologic specialists to formulate a plan of care improves care and patient outcomes, there have been no data, to our knowledge, to determine how frequently this occurs in the United States breast cancer patient. It consequently has remained unclear whether MDC, defined in this manner, is currently standard practice or whether facilities not engaging in such coordinated care are the norm. We were surprised to find that the use of MDC, defined here as a preoperative visit after breast cancer diagnosis with a surgeon, medical oncologist and radiation oncologist, was rare and occurred in only $2.8 \%$ of the United States Medicare beneficiary population overall. We were gratified to see, however, that the rates of MDC increased by over ten-fold during the period of study in question.

Although MDC is highly regarded as an optimal component of care[23], it remains difficult from our findings to suggest that it is currently standard practice in the U.S. with fewer than $3 \%$ of breast cancer Medicare patients receiving care coordinated in this fashion. Same-day consultations for all three specialties, while convenient for the patient, are even rarer with fewer than $1 \%$ of patients having that opportunity after diagnosis. This latter type of scheduling coordination requires substantial effort, and so while it was expected that a minority of patients received $\mathrm{MDC}$ and $\mathrm{MDC}_{\mathrm{SD}}$, the remarkably small proportion was surprising.

Increasingly sophisticated treatment paradigms require systems that better coordinate care among subspecialists. Changes in management decisions,[6] surgical choice,[3] patient 
satisfaction,[24] radiographic/pathologic interpretation, [8] and expedited treatment times, [9] have all been associated with the use of MDC in single-institution studies of breast cancer management. As such, the European Partnership for Action Against Cancer (EPAAC) issued a policy statement in 2014 identifying multidisciplinary teams as a core component of cancer care organizations, and defined the elements necessary for a multidisciplinary approach to cancer care in Europe.[23] In the United States, The National Accreditation for Breast Centers (NAPBC) was officially launched in 2008 to define standards for breast centers and also recognizes interdisciplinary team management as a standard component for breast centers.[12, 25]

The use of MDC in this study was not uniformly distributed, and its use varied most widely according to geographic region, with a lower likelihood of receiving $\mathrm{MDC}$ and $\mathrm{MDC}_{\mathrm{SD}}$ as population density decreased. These findings may be, in part, due to disparities in resource allocation, especially among rural locations; however the SEER-Medicare dataset cannot distinguish the institution type caring for the patient, such as a cancer center, academic, or private institution, to determine the settings in which these MDC patterns occurred. We hypothesize that institutions having greater resources and numbers of employed physicians, such as large private and academic centers, are more likely to utilize MDC, explaining these geographic differences. Interestingly, disparities in race also appear to exist in MDC utilization, with blacks receiving MDC more frequently, possibly as a result of an inner city distribution of and proximity to larger centers that may provide it.

Patients undergoing MDC experienced longer mean times from diagnosis to surgery in our series consistent with a previous analysis documenting an associated delay of 7.9 days from diagnosis to surgery for an additional consultation beyond surgical evaluation.[13] The delays observed are likely related to increased time required to coordinate provider visits. $\mathrm{MDC}_{\mathrm{SD}}$ may be one mechanism by which delays are minimized and patient convenience is maximized, although the small increases in time to surgery, even when patients are seen on different days, should not itself affect outcomes.[26] Beyond convenience, the most important influence that MDC may have on treatment is an improvement in the adherence to standard therapy, which may influences outcomes.

We noted significant variation in practice patterns associated with the use of MDC, and greater compliance with most of the quality measures in the MDC and $\mathrm{MDC}_{\mathrm{SD}}$ patients. While some did not reach statistical significance (such as post-mastectomy radiotherapy in $\mathrm{MDC}_{\mathrm{SD}}$ ) these may have been underpowered. For the radiotherapy and chemotherapy standards, greater adherence to standard practice may be due to a correlation between provider knowledge and the institutions implementing MDC, but this also may result from improved communication about patient specifics resulting from this multidisciplinary team approach[27] in which formal consultations are utilized.

When evaluating breast cancer outcomes directly, MDC was associated with modest improvements in overall and breast cancer-specific survival which did not reach statistical significance. Similarly, $\mathrm{MDC}_{\mathrm{SD}}$ was associated with a trend towards improved overall survival without a difference in breast cancer-specific mortality. The benefits of modern adjuvant therapy among breast cancer patients are well described in the literature.[1,2] 
Since our propensity score models included both baseline tumor information and treatment variables (i.e. surgery type, adjuvant chemotherapy and radiation therapy use), it seems likely that the major benefits of MDC lie, in part, within the appropriate use of adjuvant therapy, as expected.

Some limitations should inform the interpretation of our data. Our data applies to patients evaluated preoperatively in a multidisciplinary setting. Our definition of MDC may underestimate the true degree to which MDC occurs across the United States. Expanding the definition of MDC to care given by multiple specialties preoperatively and postoperatively would increase the proportion of patients felt to receive it; however, our definition represents a paradigm that is felt to provide patients with information up front and potentially improve coordination of treatment. We also defined MDC according to claims data for consultations with providers, and consultations do not necessarily equate to coordination of care, especially if performed in different health systems, which cannot be discerned via our dataset. Tumor board meetings and conferences are another way to provide preoperative MDC which is not captured in Medicare claims data, and such conferences may still provide input which can elevate care towards that which occurs in a preoperative formal consultation setting. We were also not able to assess whether MDC affects diagnostic accuracy, such as the ability to detect inflammatory or metastatic cases. The quality metrics used in our analysis may not be applicable to some patients who are of advanced age or have significant comorbidities, although we performed a sensitivity analysis excluding those over 80 and found no differences in our results. The strengths of our study exist in the large, nationally representative sample, and the granularity provided by Medicare claims, with this being the first study to our knowledge to assess MDC in the United States. Further study could be directed at the patient population receiving neoadjuvant chemotherapy, who were excluded in our analysis for homogeneity.

In summary, although the rates of MDC have increased linearly over time, very few Medicare patients with localized breast cancer received it. Implementation of MDC varied most widely according to geographic location and population density. The practice of MDC was associated with improved adherence to quality measures, including appropriate administration of adjuvant chemotherapy and radiation therapy following breast conserving surgery and mastectomy, and the modest improvements in outcomes were likely related to the appropriate administration of adjuvant therapy. Further efforts to emphasize MDC should be pursued and there should be an effort to improve multidisciplinary care across the United States in a breast cancer setting.

\section{Supplementary Material}

Refer to Web version on PubMed Central for supplementary material.

\section{Acknowledgments}

Funding: This work was supported by United States Public Health Services grant P30 CA006927 for analysis of the data via support of our biostatistics facility, and by generous private donor support from the Marlyn Fein Chapter of the Fox Chase Cancer Center Board of Associates, for analysis and interpretation of the data. 
This work was supported by United States Public Health Services grant P30 CA006927 for analysis of the data via support of our biostatistics facility, and by generous private donor support from the Marlyn Fein Chapter of the Fox Chase Cancer Center Board of Associates, for analysis and interpretation of the data.

This study used the linked SEER-Medicare database. The interpretation and reporting of these data are the sole responsibility of the authors. The authors acknowledge the efforts of the Applied Research Program, NCI; the Office of Research, Development and Information, CMS; Information Management Services (IMS), Inc.; and the Surveillance, Epidemiology, and End Results (SEER) Program tumor registries in the creation of the SEERMedicare database.

The collection of the California cancer incidence data used in this study was supported by the California Department of Public Health as part of the statewide cancer reporting program mandated by California Health and Safety Code Section 103885; the National Cancer Institute's Surveillance, Epidemiology and End Results Program under contract N01-PC-35136 awarded to the Northern California Cancer Center, contract N01-PC-35139 awarded to the University of Southern California, and contract N02-PC-15105 awarded to the Public Health Institute; and the Centers for Disease Control and Prevention's National Program of Cancer Registries, under agreement \#U55/ CCR921930-02 awarded to the Public Health Institute. The ideas and opinions expressed herein are those of the author(s) and endorsement by the State of California, Department of Public Health the National Cancer Institute, and the Centers for Disease Control and Prevention or their Contractors and Subcontractors is not intended nor should be inferred.

The authors would like to thank Yanqun Dong, M.D., Ph.D. for feedback during manuscript preparation.

\section{References}

1. Peto R, Davies C, et al. Early Breast Cancer Trialists' Collaborative Group (EBCTCG). Comparisons between different polychemotherapy regimens for early breast cancer: meta-analyses of long-term outcome among 100,000 women in 123 randomised trials. Lancet Lond Engl. 2012; 379:432-444. DOI: 10.1016/S0140-6736(11)61625-5

2. McGale P, Taylor C, et al. EBCTCG (Early Breast Cancer Trialists' Collaborative Group). Effect of radiotherapy after mastectomy and axillary surgery on 10-year recurrence and 20-year breast cancer mortality: meta-analysis of individual patient data for 8135 women in 22 randomised trials. Lancet Lond Engl. 2014; 383:2127-2135. DOI: 10.1016/S0140-6736(14)60488-8

3. Baldwin L-M, Taplin SH, Friedman H, Moe R. Access to multidisciplinary cancer care: is it linked to the use of breast-conserving surgery with radiation for early-stage breast carcinoma? Cancer. 2004; 100:701-709. DOI: 10.1002/cncr.20030 [PubMed: 14770424]

4. Lamb BW, Brown KF, Nagpal K, et al. Quality of care management decisions by multidisciplinary cancer teams: a systematic review. Ann Surg Oncol. 2011; 18:2116-2125. DOI: 10.1245/ s10434-011-1675-6 [PubMed: 21442345]

5. Gomella LG, Lin J, Hoffman-Censits J, et al. Enhancing prostate cancer care through the multidisciplinary clinic approach: a 15-year experience. J Oncol Pract Am Soc Clin Oncol. 2010; 6:e5-e10. DOI: 10.1200/JOP.2010.000071

6. Chang JH, Vines E, Bertsch H, et al. The impact of a multidisciplinary breast cancer center on recommendations for patient management: the University of Pennsylvania experience. Cancer. 2001; 91:1231-1237. [PubMed: 11283921]

7. Korman H, Lanni T, Shah C, et al. Impact of a prostate multidisciplinary clinic program on patient treatment decisions and on adherence to NCCN guidelines: the William Beaumont Hospital experience. Am J Clin Oncol. 2013; 36:121-125. DOI: 10.1097/COC.0b013e318243708f [PubMed: 22307214]

8. Newman EA, Guest AB, Helvie MA, et al. Changes in surgical management resulting from case review at a breast cancer multidisciplinary tumor board. Cancer. 2006; 107:2346-2351. DOI: 10.1002/cncr.22266 [PubMed: 16998942]

9. Gabel M, Hilton NE, Nathanson SD. Multidisciplinary breast cancer clinics. Do they work? Cancer. 1997; 79:2380-2384. [PubMed: 9191526]

10. Devitt B, Philip J, McLachlan S-A. Team dynamics, decision making, and attitudes toward multidisciplinary cancer meetings: health professionals' perspectives. J Oncol Pract Am Soc Clin Oncol. 2010; 6:e17-20. DOI: 10.1200/JOP.2010.000023 
11. Bunnell CA, Weingart SN, Swanson S, et al. Models of multidisciplinary cancer care: physician and patient perceptions in a comprehensive cancer center. J Oncol Pract Am Soc Clin Oncol. 2010; 6:283-288. DOI: $10.1200 /$ JOP.2010.000138

12. American College of Surgeons. Natl Accreditation Breast Cent Stand Man. Interdisciplinary Patient Management Section 2.1.

13. Bleicher RJ, Ruth K, Sigurdson ER, et al. Preoperative delays in the US Medicare population with breast cancer. J Clin Oncol Off J Am Soc Clin Oncol. 2012; 30:4485-4492. DOI: 10.1200/JCO. 2012.41.7972

14. CoC Quality of Care Measures. [Accessed 27 Sep 2015] Am Coll Surg. https://www.facs.org/ quality-programs/cancer/ncdb/qualitymeasures

15. [Accessed 27 Sep 2015] NAPBC Standards. Am Coll Surg. https://www.facs.org/quality-programs/ napbc/standards

16. Lunceford JK, Davidian M. Stratification and weighting via the propensity score in estimation of causal treatment effects: a comparative study. Stat Med. 2004; 23:2937-2960. DOI: 10.1002/sim. 1903 [PubMed: 15351954]

17. Robins JM, Hernán MA, Brumback B. Marginal structural models and causal inference in epidemiology. Epidemiol Camb Mass. 2000; 11:550-560.

18. Elixhauser A, Steiner C, Harris DR, Coffey RM. Comorbidity measures for use with administrative data. Med Care. 1998; 36:8-27. [PubMed: 9431328]

19. van Walraven C, Austin PC, Jennings A, et al. A modification of the Elixhauser comorbidity measures into a point system for hospital death using administrative data. Med Care. 2009; 47:626-633. DOI: 10.1097/MLR.0b013e31819432e5 [PubMed: 19433995]

20. Charlson ME, Pompei P, Ales KL, MacKenzie CR. A new method of classifying prognostic comorbidity in longitudinal studies: development and validation. J Chronic Dis. 1987; 40:373-383. [PubMed: 3558716]

21. Fine JP, Gray RJ. A Proportional Hazards Model for the Subdistribution of a Competing Risk. J Am Stat Assoc. 1999; 94:496-509. DOI: 10.2307/2670170

22. FEH. Regres Model Strateg. Springer; New York: 2001. General Aspects of Fitting Regression Models; p. 11-40.

23. Borras JM, Albreht T, et al. European Partnership Action Against Cancer consensus group. Policy statement on multidisciplinary cancer care. Eur J Cancer Oxf Engl 1990. 2014; 50:475-480. DOI: 10.1016/j.ejca.2013.11.012

24. Komatsu H, Nakayama K, Togari T, et al. Information sharing and case conference among the multidisciplinary team improve patients' perceptions of care. Open Nurs J. 2011; 5:79-85. DOI: 10.2174/1874434601105010079 [PubMed: 22135715]

25. Bensenhaver J, Winchester DP. Surgical leadership and standardization of multidisciplinary breast cancer care: the evolution of the National Accreditation Program for Breast Centers. Surg Oncol Clin N Am. 2014; 23:609-616. DOI: 10.1016/j.soc.2014.03.005 [PubMed: 24882354]

26. Bleicher RJ, Ruth K, Sigurdson ER, et al. Time to Surgery and Breast Cancer Survival in the United States. JAMA Oncol. 2016; 2:330-339. DOI: 10.1001/jamaoncol.2015.4508 [PubMed: 26659430]

27. Aizer AA, Paly JJ, Efstathiou JA. Multidisciplinary care and management selection in prostate cancer. Semin Radiat Oncol. 2013; 23:157-164. DOI: 10.1016/j.semradonc.2013.01.001 [PubMed: 23763881] 


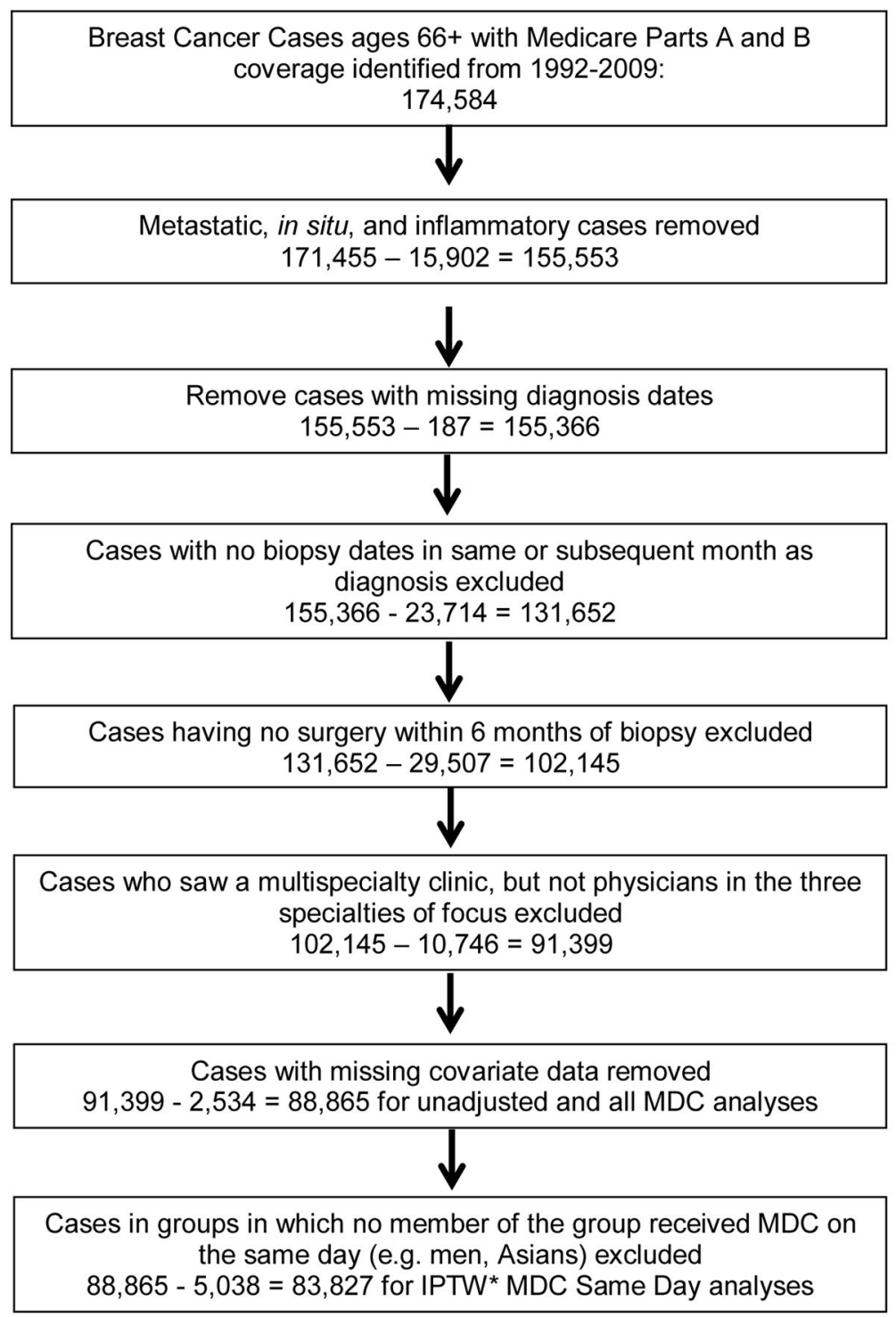

Figure 1.

Exclusion criteria. *IPTW=inverse probability of treatment weights. 


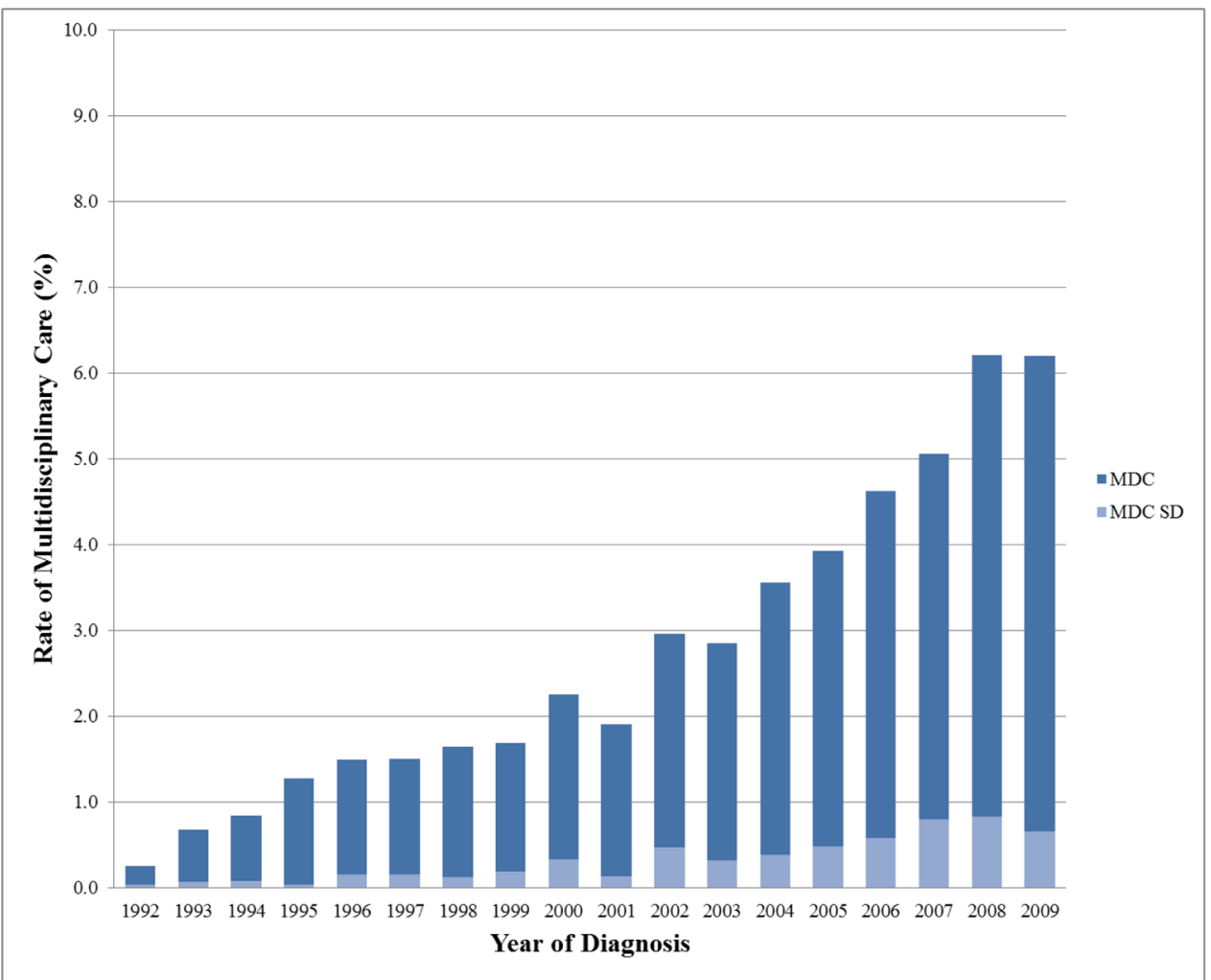

Figure 2.

Rates of multidisciplinary care over time among Medicare patients having invasive nonmetastatic, non-inflammatory breast cancer in the United States. MDC $=$ all patients receiving multidisciplinary care. $\mathrm{MDC}_{\mathrm{SD}}=$ subset of patients receiving $\mathrm{MDC}$ on the same date. 


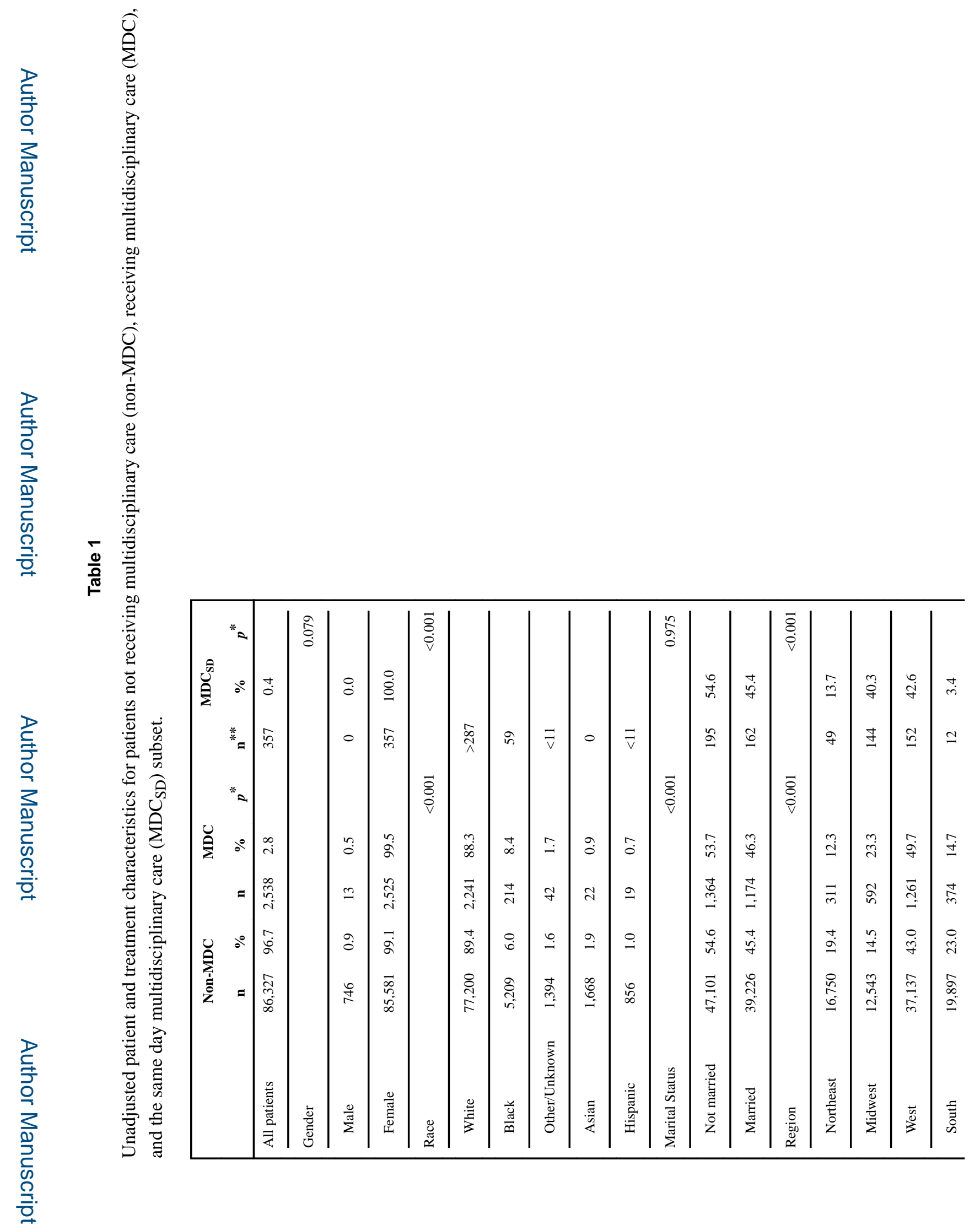

Breast Cancer Res Treat. Author manuscript; available in PMC 2017 November 01. 


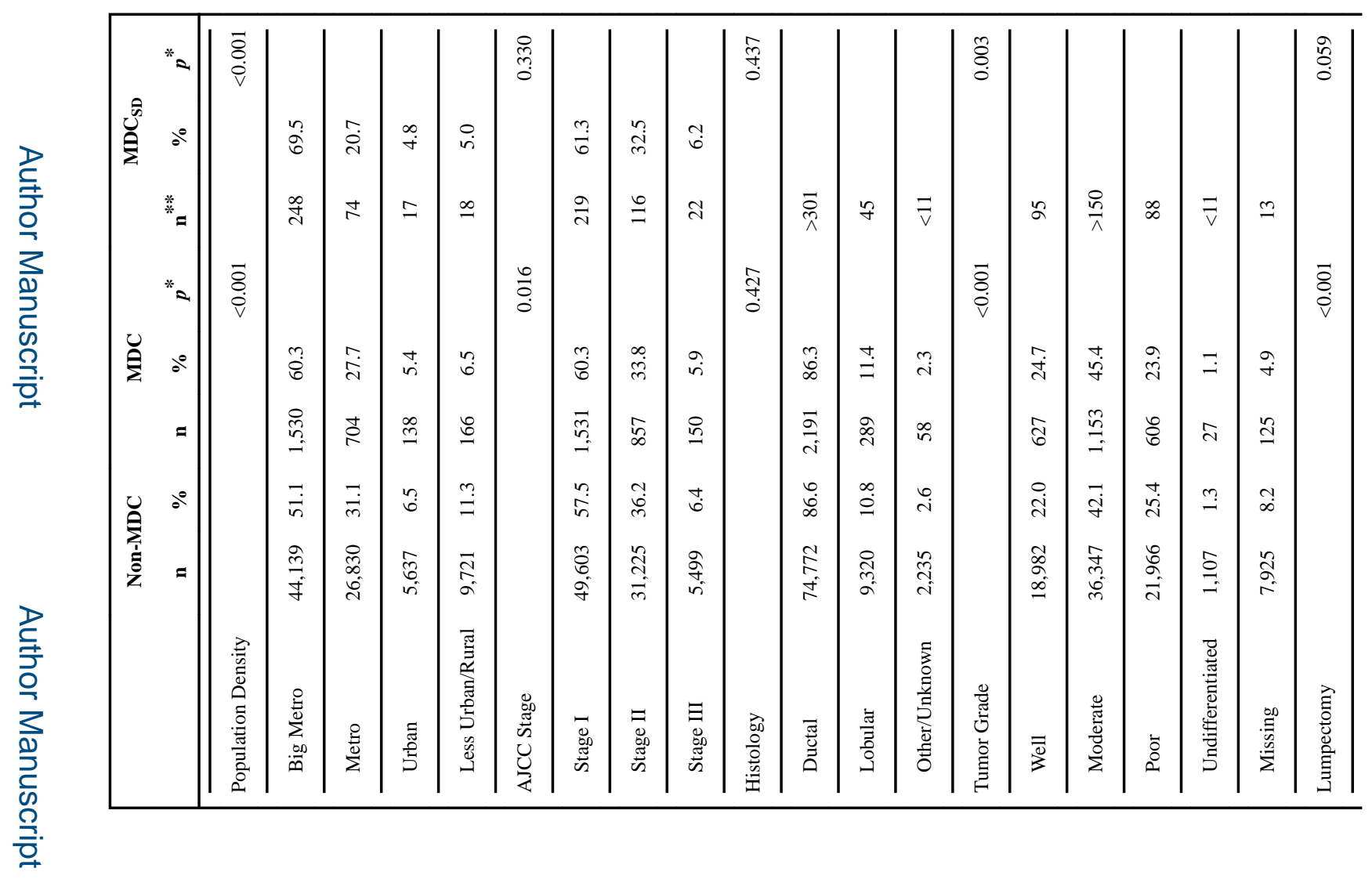

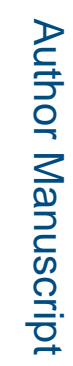

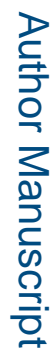




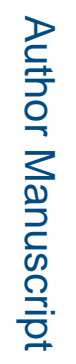

I

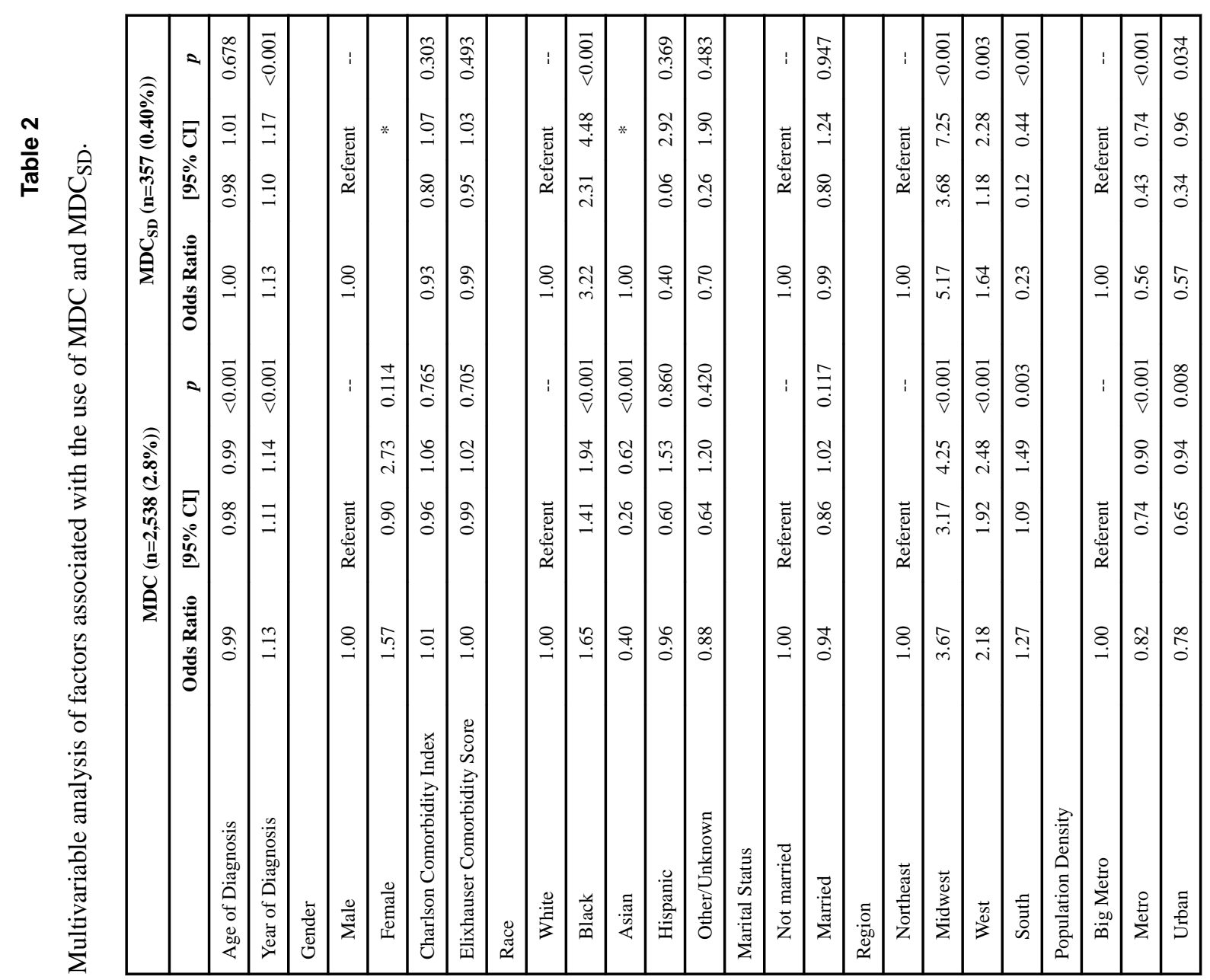

Breast Cancer Res Treat. Author manuscript; available in PMC 2017 November 01. 


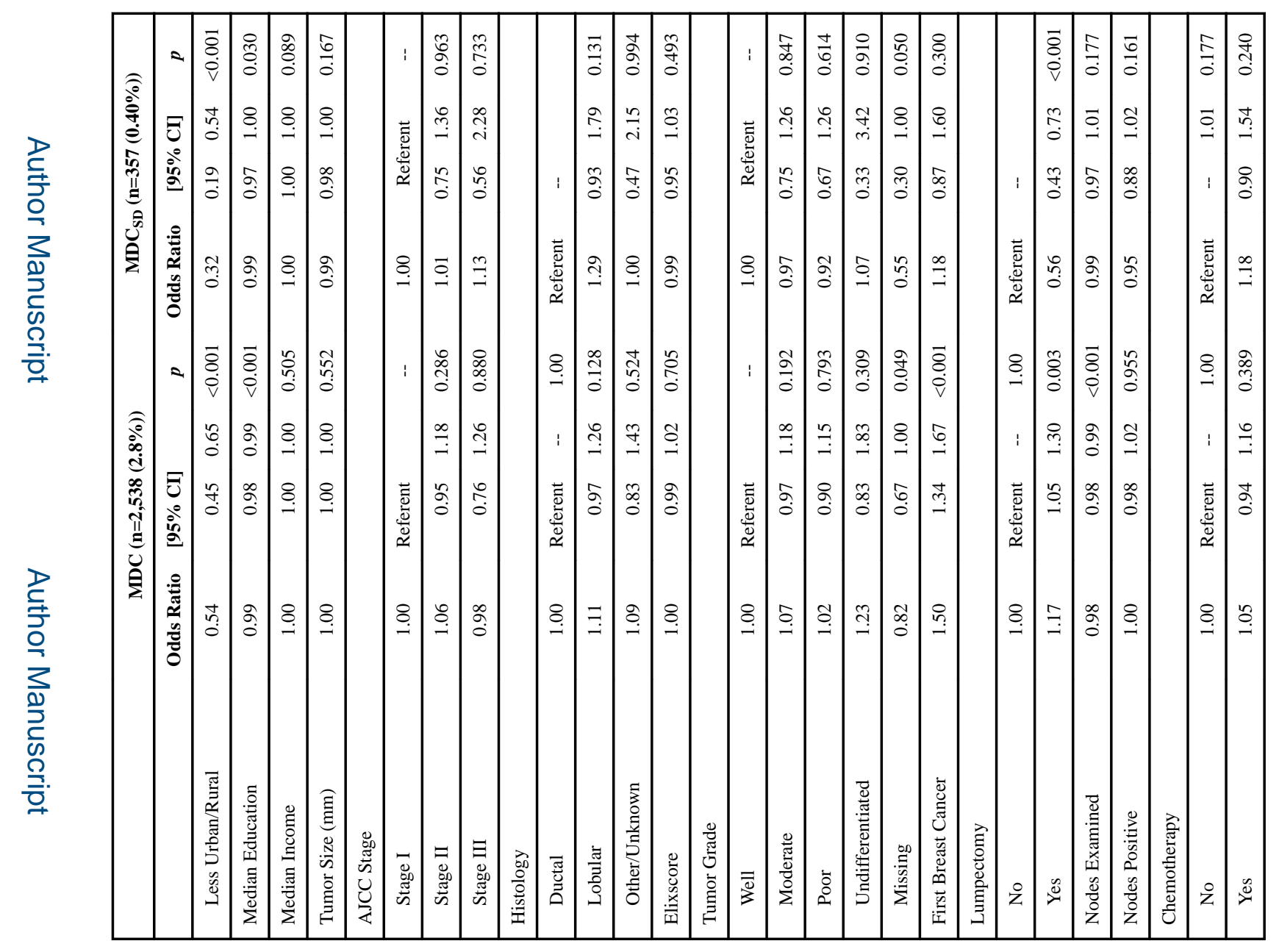

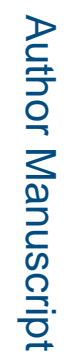

로을 


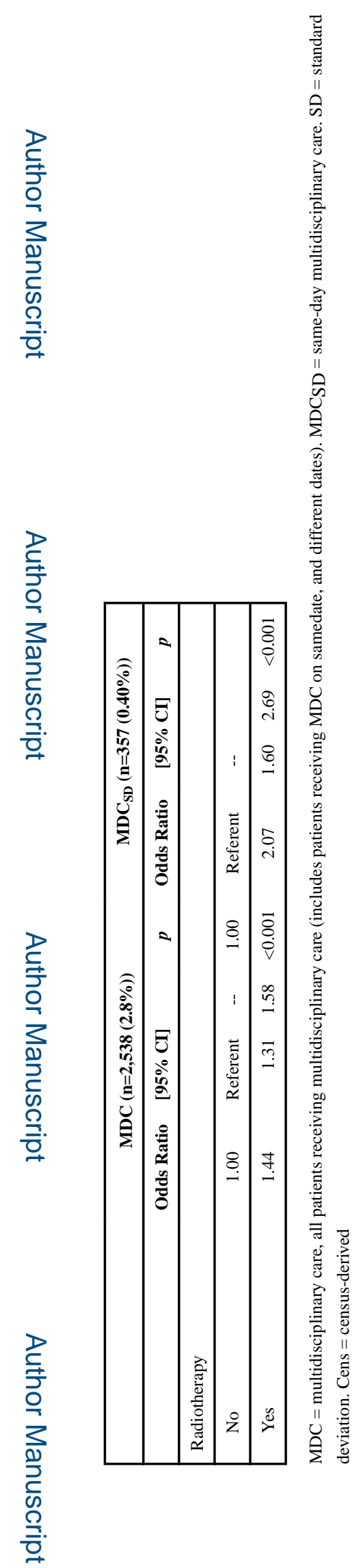

Breast Cancer Res Treat. Author manuscript; available in PMC 2017 November 01. 


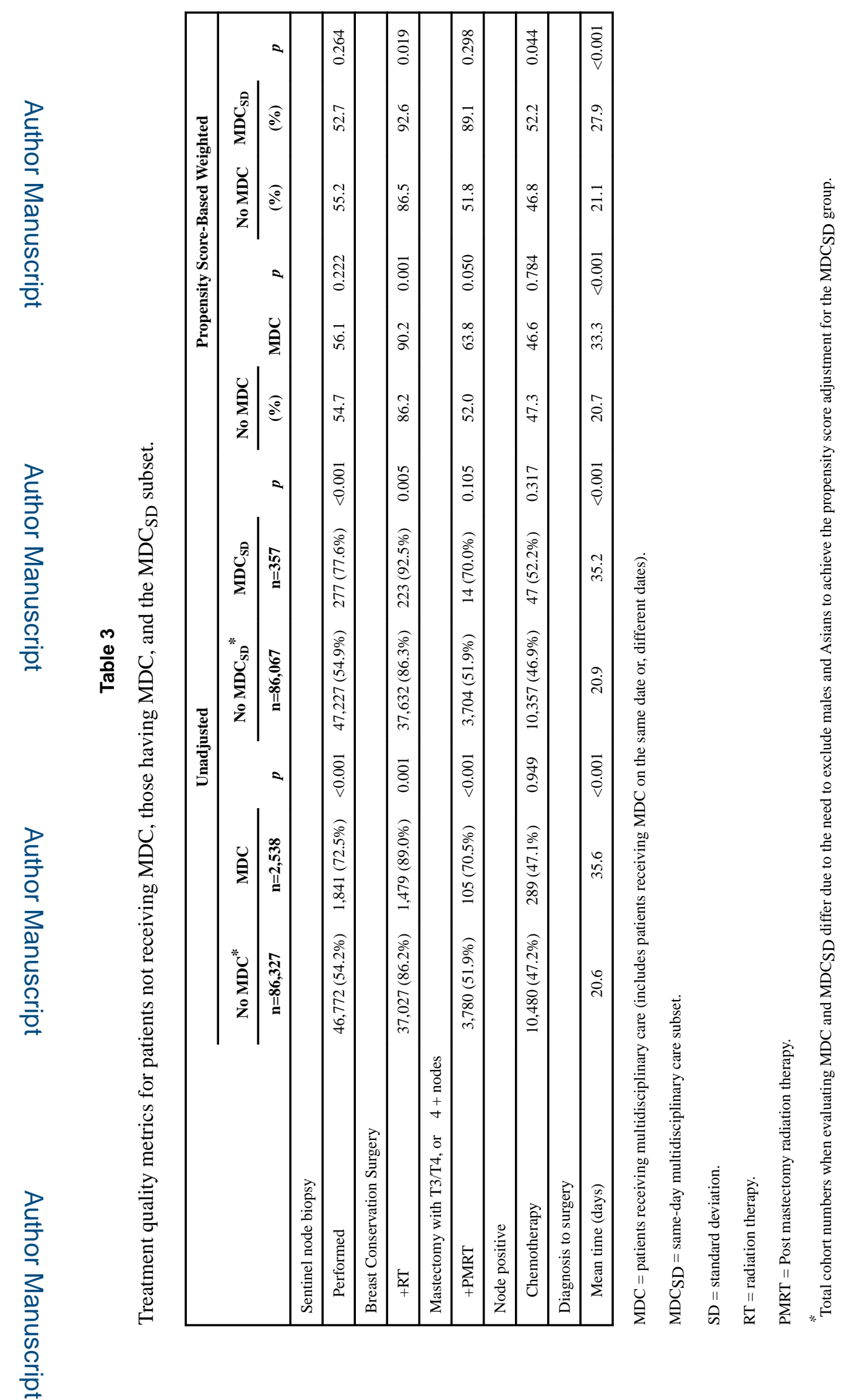

Breast Cancer Res Treat. Author manuscript; available in PMC 2017 November 01. 